\title{
An Efficient Family of Optimal Eighth-Order Iterative Methods for Solving Nonlinear Equations and Its Dynamics
}

\author{
Anuradha Singh and J. P. Jaiswal \\ Department of Mathematics, Maulana Azad National Institute of Technology, Bhopal, Madhya Pradesh 462051, India \\ Correspondence should be addressed to J. P. Jaiswal; asstprofjpmanit@gmail.com
}

Received 2 May 2014; Revised 7 August 2014; Accepted 28 August 2014; Published 15 September 2014

Academic Editor: Herbert Homeier

Copyright ( 2014 A. Singh and J. P. Jaiswal. This is an open access article distributed under the Creative Commons Attribution License, which permits unrestricted use, distribution, and reproduction in any medium, provided the original work is properly cited.

The prime objective of this paper is to design a new family of optimal eighth-order iterative methods by accelerating the order of convergence of the existing seventh-order method without using more evaluations for finding simple root of nonlinear equations. Numerical comparisons have been carried out to demonstrate the efficiency and performance of the proposed method. Finally, we have compared new method with some existing eighth-order methods by basins of attraction and observed that the proposed scheme is more efficient.

\section{Introduction}

One of the prominent iterative methods for finding simple roots of a nonlinear equation, $f(x)=0$, is Newton's method which is described as follows:

$$
x_{n+1}=x_{n}-\frac{f\left(x_{n}\right)}{f^{\prime}\left(x_{n}\right)} .
$$

It is well known that the order of convergence of Newton's method is two. Solving a nonlinear equation is one of the most important and challenging tasks in numerical analysis. The vast literature is available for computing the solution of nonlinear equations or system of nonlinear equations; for instance, one can see [1-7]. During the last few years, multipoint methods have drawn the attention of many researchers. In [5] Petkovic et al. have presented a large collection of with and without memory multipoint methods for solving nonlinear equations. In the recent past, researchers have focused to optimize the existing methods without additional evaluation of function and derivative. In [8] Chun et al. have introduced the method of choosing weight functions in iterative methods for simple root. Recently, Soleymani and Mousavi [9] introduced the following seventh-order method:

$$
\begin{gathered}
y_{n}=x_{n}-\frac{f\left(x_{n}\right)}{f^{\prime}\left(x_{n}\right)}, \\
z_{n}=y_{n}-\frac{f\left(y_{n}\right)}{f\left[x_{n}, y_{n}\right]} \cdot G\left(t_{n}\right), \\
x_{n+1}=z_{n}-\frac{f\left(z_{n}\right)}{f\left[y_{n}, z_{n}\right]} \cdot H\left(t_{n}\right),
\end{gathered}
$$

where $t_{n}=f\left(y_{n}\right) / f\left(x_{n}\right)$ and $G(0)=G^{\prime}(0)=1,\left|G^{\prime \prime}(0)\right|<$ $+\infty ; H(0)=1, H^{\prime}(0)=0, H^{\prime \prime}(0)=2,\left|H^{(3)}(0)\right|<+\infty$. To compare the efficiency of different iterative methods the efficiency index is defined by $p^{1 / n}$, where $p$ is the order of convergence and $n$ is the number of total function and derivative evaluations per iteration [7]. According to the conjecture of optimality, the optimal order of any multipoint iterative method without memory is given by $2^{n-1}$ where $n$ is the total number of function evaluations [4]. Thus, the efficiency index of the method $(2)$ is $7^{1 / 4} \approx 1.626$. Also, this method is not optimal, because this method requires four evaluations (three functions and one derivative) and for 
TABLE 1: Functions and their roots.

\begin{tabular}{lc}
\hline Function $(f(x))$ & $\operatorname{Root}(\alpha)$ \\
\hline$f_{1}(x)=10 x e^{-x^{2}}-1$ & $\alpha_{1} \approx 1.67963 \ldots$ \\
$f_{2}(x)=x^{5}+x^{4}+4 x^{2}-15$ & $\alpha_{2} \approx 1.34742 \ldots$ \\
$f_{3}(x)=x e^{x^{2}}-(\sin x)^{2}+3 \cos x+5$ & $\alpha_{3} \approx-1.20764 \ldots$ \\
$f_{4}(x)=x^{4}+\sin \left(\frac{\pi}{x^{2}}\right)-5$ & $\alpha_{4}=\sqrt{2}$ \\
$f_{5}(x)=x^{2} e^{x}-\sin x$ & $\alpha_{5}=0$ \\
$f_{6}(x)=\left(\sin x-\frac{\sqrt{2}}{2}\right)^{2}(x+1)$ & $\alpha_{6}=-1$ \\
$f_{7}(x)=\sin 3 x+x \cos x$ & $\alpha_{7} \approx 1.19776 \ldots$ \\
\hline
\end{tabular}

optimal its order should be $2^{3}=8$. The aim of this paper is to accelerate the order of convergence of the method (2) from seven to eight without adding more evaluations. Thus, it will agree with Kung-Traub conjecture as well as has higher efficiency index.

The rest of the paper is organized as follows: in Section 2, we propose a new family of optimal eighth-order iterative method for finding simple root of nonlinear equations. In Section 3, we employ some numerical examples to compare the performance of our new method with some existing eighth-order methods. Section 4 is devoted to the dynamical comparisons by basins of attraction in the complex plane of our proposed method with some existing methods. Finally, in the last section we give the conclusion.

\section{Improved Scheme and Convergence Analysis}

In this section, the order of convergence of the method (2) will be improved. The order of convergence of the method (2) is seven by using four evaluations [ $f\left(x_{n}\right), f^{\prime}\left(x_{n}\right), f\left(y_{n}\right)$, $f\left(z_{n}\right)$, which is clearly not optimal. To build an optimal eighth-order method without using more evaluations, we consider

$$
\begin{gathered}
y_{n}=x_{n}-A\left(t_{1}\right) \cdot \frac{f\left(x_{n}\right)}{f^{\prime}\left(x_{n}\right)}, \\
z_{n}=y_{n}-B\left(t_{2}\right) \cdot \frac{f\left(y_{n}\right)}{f\left[x_{n}, y_{n}\right]}, \\
x_{n+1}=z_{n}-\left\{P\left(t_{2}\right)+Q\left(t_{3}\right)+R\left(t_{4}\right)\right\} \cdot \frac{f\left(z_{n}\right)}{f\left[y_{n}, z_{n}\right]},
\end{gathered}
$$

where $t_{1}=f\left(x_{n}\right) / f^{\prime}\left(x_{n}\right), t_{2}=f\left(y_{n}\right) / f\left(x_{n}\right), t_{3}=f\left(z_{n}\right) /$ $f\left(y_{n}\right)$, and $t_{4}=f\left(z_{n}\right) / f\left(x_{n}\right)$. The weight functions should be chosen such that the order arrives at optimal level eight. Theorem 1 gives the conditions on weight functions to reach at the optimal order of convergence.

Theorem 1. Let the function $f: D \subseteq \Re \rightarrow \Re$ has sufficient number of continuous derivatives in a neighborhood $D$ of simple root $\alpha$ of $f$. Then the method described by (3) has eighth-order convergence, provided the weight functions $A\left(t_{1}\right)$, $B\left(t_{2}\right), P\left(t_{2}\right), Q\left(t_{3}\right)$, and $R\left(t_{4}\right)$ satisfy the following conditions:

$$
\begin{gathered}
A(0)=1, \quad A^{\prime}(0)=0, \quad A^{\prime \prime}(0)=0, \quad\left|A^{(3)}(0)\right|<+\infty \\
B(0)=1, \quad B^{\prime}(0)=1, \quad\left|B^{(3)}(1)\right|<+\infty \\
R(0)=1-P(0)-Q(0) \\
P^{\prime}(0)=0, \quad P^{\prime \prime}(0)=2, \\
P^{(3)}(0)=6 B^{\prime \prime}(0)-12, \quad\left|P^{(4)}(0)\right|<+\infty \\
Q^{\prime}(0)=0, \quad\left|Q^{\prime \prime}(0)\right|<+\infty \\
R^{\prime}(0)=2, \quad\left|R^{\prime \prime}(0)\right|<+\infty .
\end{gathered}
$$

Proof. Let $e_{n}=x_{n}-\alpha$ be the error in the $n$th iterate and $c_{h}=f^{(h)}(\alpha) / h !, h=1,2,3, \ldots$. We provide Taylor's series expansion of each term involved in (3). By Taylor expansion around the simple root in the $n$th iterate, we have

$$
\begin{aligned}
& f\left(x_{n}\right)=c_{1} e_{n}+c_{2} e_{n}^{2}+\cdots+O\left(e_{n}^{10}\right), \\
& f^{\prime}\left(x_{n}\right)=c_{1}+2 c_{2} e_{n}+\cdots+O\left(e_{n}^{9}\right) .
\end{aligned}
$$

From (5) and (6), it can be easily found that

$$
\frac{f\left(x_{n}\right)}{f^{\prime}\left(x_{n}\right)}=e_{n}-\frac{c_{2} e_{n}^{2}}{c_{1}}+\cdots+O\left(e_{n}^{9}\right) .
$$

By considering this relation and $A(0)=1$, we obtain

$$
\begin{aligned}
y_{n}= & \alpha+\left\{\frac{c_{2}}{c_{1}}-A^{\prime}(0)\right\} e_{n}^{2} \\
& +\left\{-\frac{2 c_{2}^{2}}{c_{1}^{2}}+\frac{2\left(c_{3}+c_{2} A^{\prime}(0)\right)}{c_{1}}-\frac{A^{\prime \prime}(0)}{2}\right\} e_{n}^{3} \\
& +\cdots+O\left(e_{n}^{9}\right) .
\end{aligned}
$$

Taylor's expansion of $f\left(y_{n}\right)$ around the root $\alpha$ is given as

$$
\begin{aligned}
f\left(y_{n}\right)= & \left(c_{2}-c_{1} A^{\prime}(0)\right) e_{n}^{2} \\
& +\left\{-\frac{2 c_{2}^{2}}{c_{1}}+2\left(c_{3}+c_{2} A^{\prime}(0)\right)-\frac{1}{2} c_{1} A^{\prime \prime}(0)\right\} e_{n}^{3} \\
& +\cdots+O\left(e_{n}^{9}\right) .
\end{aligned}
$$


TABLE 2: Comparison of absolute value of the functions after third iteration.

\begin{tabular}{|c|c|c|c|c|c|c|c|c|}
\hline$|f|$ & Guess & $M_{8,1}$ & $M_{8,2}$ & $M_{8,3}$ & $M_{8,4}$ & $M_{8,5}$ & $M_{8,6}$ & $O M_{8,4}$ \\
\hline \multirow{4}{*}{$\left|f_{1}\right|$} & 1.72 & $0.2 e-617$ & $0.2 e-617$ & $0.2 e-636$ & $0.2 e-602$ & $0.1 e-660$ & $0.2 e-654$ & $0.4 e-688$ \\
\hline & 1.5 & $0.1 e-357$ & $0.7 e-358$ & $0.7 e-365$ & $0.3 e-346$ & $0.2 e-375$ & $0.8 e-361$ & $0.2 e-448$ \\
\hline & 1.7 & $0.5 e-796$ & $0.5 e-796$ & $0.1 e-794$ & $0.2 e-762$ & $0.4 e-828$ & $0.5 e-809$ & $0.4 e-866$ \\
\hline & 1.1 & $0.8 e-259$ & $0.7 e-257$ & $0.3 e-179$ & $0.6 e-175$ & $0.1 e-205$ & $0.2 e-204$ & $0.6 e-259$ \\
\hline \multirow{4}{*}{$\left|f_{2}\right|$} & 1.1 & $\mathrm{NC}$ & $0.6 e-52$ & $0.1 e-177$ & $0.9 e-116$ & $0.7 e-165$ & $0.5 e-299$ & $0.3 e-127$ \\
\hline & 1.8 & $0.3 e-148$ & $0.2 e-149$ & $0.5 e-194$ & $0.2 e-175$ & $0.1 e-195$ & $0.4 e-187$ & $0.1 e-225$ \\
\hline & 1.5 & $0.6 e-347$ & $0.6 e-347$ & $0.2 e-377$ & $0.1 e-348$ & $0.9 e-437$ & $0.1 e-390$ & $0.4 e-436$ \\
\hline & 2.0 & $0.5 e-97$ & $0.6 e-99$ & $0.3 e-148$ & $0.1 e-133$ & $0.3 e-134$ & $0.4 e-132$ & $0.1 e-150$ \\
\hline \multirow{4}{*}{$\left|f_{3}\right|$} & -1.1 & $0.5 e-234$ & $0.1 e-235$ & $0.4 e-337$ & $0.8 e-285$ & $0.1 e-325$ & $0.6 e-433$ & $0.1 e-301$ \\
\hline & -1.5 & $0.5 e-124$ & $0.7 e-125$ & $0.2 e-182$ & $0.2 e-161$ & $0.2 e-253$ & $0.2 e-205$ & $0.2 e-254$ \\
\hline & -1.0 & div. & $0.2 e-45$ & $0.1 e-173$ & $0.2 e-107$ & $0.3 e-158$ & $0.3 e-254$ & $0.9 e-116$ \\
\hline & -1.3 & $0.1 e-383$ & $0.1 e-383$ & $0.6 e-404$ & $0.2 e-370$ & $0.2 e-411$ & $0.3 e-460$ & $0.1 e-468$ \\
\hline \multirow{4}{*}{$\left|f_{4}\right|$} & 1.0 & $0.9 e-255$ & $0.1 e-251$ & $0.2 e-226$ & $0.3 e-223$ & $0.2 e-199$ & $0.5 e-227$ & $0.2 e-262$ \\
\hline & 1.6 & $0.8 e-338$ & $0.7 e-338$ & $0.9 e-356$ & $0.2 e-329$ & $0.3 e-370$ & $0.5 e-430$ & $0.1 e-441$ \\
\hline & 1.5 & $0.2 e-508$ & $0.2 e-508$ & $0.1 e-519$ & $0.1 e-487$ & $0.9 e-526$ & $0.4 e-560$ & $0.3 e-532$ \\
\hline & 2.1 & $0.1 e-105$ & $0.2 e-107$ & $0.1 e-146$ & $0.9 e-134$ & $0.1 e-144$ & $0.1 e-143$ & $0.3 e-159$ \\
\hline \multirow{4}{*}{$\left|f_{5}\right|$} & 0.1 & $0.1 e-272$ & $0.3 e-273$ & $0.1 e-340$ & $0.4 e-303$ & $0.6 e-349$ & $0.1 e-338$ & $0.1 e-364$ \\
\hline & 0.5 & $0.5 e-264$ & $0.8 e-265$ & $0.1 e-346$ & $0.3 e-301$ & $0.2 e-342$ & $0.7 e-382$ & $0.5 e-339$ \\
\hline & -0.1 & $0.4 e-475$ & $0.1 e-475$ & $0.2 e-470$ & $0.1 e-455$ & $0.3 e-441$ & $0.2 e-436$ & $0.4 e-485$ \\
\hline & -0.5 & $0.1 e-270$ & $0.3 e-270$ & $0.8 e-237$ & $0.2 e-233$ & $0.1 e-235$ & $0.3 e-233$ & $0.2 e-277$ \\
\hline \multirow{4}{*}{$\left|f_{6}\right|$} & -0.8 & $0.3 e-158$ & $0.1 e-162$ & $0.4 e-258$ & $0.9 e-214$ & $0.4 e-248$ & $0.6 e-288$ & $0.3 e-254$ \\
\hline & -1.2 & $0.3 e-422$ & $0.4 e-423$ & $0.7 e-398$ & $0.9 e-387$ & $0.3 e-385$ & $0.1 e-380$ & $0.2 e-435$ \\
\hline & -0.9 & $0.1 e-425$ & $0.1 e-425$ & $0.1 e-456$ & $0.1 e-424$ & $0.4 e-500$ & $0.4 e-457$ & $0.6 e-526$ \\
\hline & -1.5 & $0.1 e-324$ & $0.8 e-324$ & $0.7 e-262$ & $0.1 e-258$ & $0.7 e-273$ & $0.1 e-272$ & $0.3 e-336$ \\
\hline \multirow{4}{*}{$\left|f_{7}\right|$} & 1.0 & $0.1 e-446$ & $0.2 e-448$ & $0.1 e-374$ & $0.1 e-371$ & $0.2 e-378$ & $0.7 e-374$ & $0.2 e-505$ \\
\hline & 0.8 & $0.6 e-81$ & NC & $0.3 e-130$ & $0.4 e-114$ & $0.7 e-159$ & $0.1 e-116$ & $0.6 e-150$ \\
\hline & 1.8 & $\mathrm{NC}$ & $\mathrm{NC}$ & $0.2 e-38$ & $0.3 e-20$ & $\mathrm{NC}$ & $\mathrm{NC}$ & $0.6 e-29$ \\
\hline & 0.3 & $0.1 e-226$ & $0.3 e-235$ & $0.8 e-244$ & $0.7 e-215$ & $0.7 e-277$ & $0.1 e-278$ & $0.2 e-416$ \\
\hline
\end{tabular}

Here div. $=$ divergent $\mathrm{NC}=$ not convergent.

In view of (5) and (9), we get

$$
\begin{aligned}
\frac{f\left(y_{n}\right)}{f\left(x_{n}\right)}= & \left\{\frac{c_{2}}{c_{1}}-A^{\prime}(0)\right\} e_{n} \\
& +\left\{-\frac{3 c_{2}^{2}}{c_{1}^{2}}+\frac{2 c_{3}+3 c_{2} A^{\prime}(0)}{c_{1}}-\frac{A^{\prime \prime}(0)}{2}\right\} e_{n}^{2} \\
& +\cdots+O\left(e_{n}^{9}\right), \\
f\left[x_{n}, y_{n}\right]= & \frac{f\left(x_{n}\right)-f\left(y_{n}\right)}{x_{n}-y_{n}}=c_{1}+c_{2} e_{n} \\
& +\left\{\frac{c_{2}^{2}}{c_{1}}+c_{3}-c_{2} A^{\prime}(0)\right\} e_{n}^{2} \\
& +\cdots+O\left(e_{n}^{9}\right) .
\end{aligned}
$$

Using (9), (10), (11) and $A^{\prime}(0)=0, B(0)=1, B^{\prime}(0)=1$, in the second step of (3), we find

$z_{n}=\alpha$

$$
\begin{aligned}
& +\frac{c_{2}\left(c_{1}\left(-2 c_{3}+c_{1} A^{\prime \prime}(0)\right)-c_{2}^{2}\left(-6+B^{\prime \prime}(0)\right)\right)}{2 c_{1}^{3}} e_{n}^{4} \\
& +\frac{1}{12 c_{1}^{4}}\left(-3 c_{1}^{2}\left(-4 c_{3}+c_{1} A^{\prime \prime}(0)\right)\left(-2 c_{3}+c_{1} A^{\prime \prime}(0)\right)\right. \\
& +3 c_{1} c_{2}^{2}\left(-4 c_{3}+c_{1} A^{\prime \prime}(0)\right) \\
& \times\left(-20+3 B^{\prime \prime}(0)\right)+2 c_{1}^{2} c_{2}\left(-12 c_{4}+c_{1} A^{(3)}(0)\right) \\
& \left.+c_{2}^{4}\left(54\left(-4+B^{\prime \prime}(0)-2 B^{(3)}(0)\right)\right)\right) e_{n}^{5} \\
& +\cdots+O\left(e_{n}^{9}\right) .
\end{aligned}
$$


TABLE 3: Comparison of number of iterations and total number of function evaluations.

\begin{tabular}{|c|c|c|c|c|c|c|c|c|}
\hline$f$ & Guess & $M_{8,1}$ & $M_{8,2}$ & $M_{8,3}$ & $M_{8,4}$ & $M_{8,5}$ & $M_{8,6}$ & $O M_{8,4}$ \\
\hline \multirow{4}{*}{$f_{1}$} & 1.72 & $2(8)$ & $2(8)$ & $2(8)$ & $2(8)$ & $2(8)$ & $2(8)$ & $2(8)$ \\
\hline & 1.5 & $3(12)$ & $3(12)$ & $3(12)$ & $3(12)$ & $3(12)$ & $3(12)$ & $2(8)$ \\
\hline & 1.7 & $2(8)$ & $2(8)$ & $2(8)$ & $2(8)$ & $2(8)$ & $2(8)$ & $2(8)$ \\
\hline & 1.1 & $3(12)$ & $3(12)$ & $3(12)$ & $3(12)$ & $3(12)$ & $3(12)$ & $2(8)$ \\
\hline \multirow{4}{*}{$f_{2}$} & 1.1 & $\mathrm{NC}$ & $3(12)$ & $3(12)$ & $3(12)$ & $3(12)$ & $3(12)$ & $3(12)$ \\
\hline & 1.8 & $3(12)$ & $3(12)$ & $3(12)$ & $3(12)$ & $3(12)$ & $3(12)$ & $3(12)$ \\
\hline & 1.5 & $3(12)$ & $3(12)$ & $3(12)$ & $3(12)$ & $2(8)$ & $3(12)$ & $2(8)$ \\
\hline & 2.0 & $3(12)$ & $3(12)$ & $3(12)$ & $3(12)$ & $3(12)$ & $3(12)$ & $3(12)$ \\
\hline \multirow{4}{*}{$f_{3}$} & -1.1 & $3(12)$ & $3(12)$ & $3(12)$ & $3(12)$ & $3(12)$ & $2(8)$ & $2(8)$ \\
\hline & -1.5 & $3(12)$ & $3(12)$ & $3(12)$ & $3(12)$ & $3(12)$ & $3(12)$ & $3(12)$ \\
\hline & -1.0 & div. & $4(16)$ & $3(12)$ & $3(12)$ & $3(12)$ & $3(12)$ & $3(12)$ \\
\hline & -1.3 & $3(12)$ & $3(12)$ & $3(12)$ & $3(12)$ & $2(8)$ & $2(8)$ & $2(8)$ \\
\hline \multirow{4}{*}{$f_{4}$} & 1.0 & $3(12)$ & $3(12)$ & $3(12)$ & $3(12)$ & $3(12)$ & $3(12)$ & $3(12)$ \\
\hline & 1.6 & $3(12)$ & $3(12)$ & $3(12)$ & $3(12)$ & $3(12)$ & $2(8)$ & $2(8)$ \\
\hline & 1.5 & $2(8)$ & $2(8)$ & $2(8)$ & $2(8)$ & $2(8)$ & $2(8)$ & $2(8)$ \\
\hline & 2.1 & $3(12)$ & $3(12)$ & $3(12)$ & $3(12)$ & $3(12)$ & $3(12)$ & $3(12)$ \\
\hline \multirow{4}{*}{$f_{5}$} & 0.1 & $3(12)$ & $3(12)$ & $3(12)$ & $3(12)$ & $3(12)$ & $3(12)$ & $3(12)$ \\
\hline & 0.5 & $3(12)$ & $3(12)$ & $3(12)$ & $3(12)$ & $3(12)$ & $3(12)$ & $3(12)$ \\
\hline & -0.1 & $2(8)$ & $2(8)$ & $2(8)$ & $2(8)$ & $2(8)$ & $2(8)$ & $2(8)$ \\
\hline & -0.5 & $3(12)$ & $3(12)$ & $3(12)$ & $3(12)$ & $3(12)$ & $3(12)$ & $3(12)$ \\
\hline \multirow{4}{*}{$f_{6}$} & -0.8 & $3(12)$ & $3(12)$ & $3(12)$ & $3(12)$ & $3(12)$ & $3(12)$ & $3(12)$ \\
\hline & -1.2 & $2(8)$ & $2(8)$ & $3(12)$ & $3(12)$ & $3(12)$ & $3(12)$ & $2(8)$ \\
\hline & -0.9 & $2(8)$ & $2(8)$ & $2(8)$ & $3(12)$ & $2(8)$ & $2(8)$ & $2(8)$ \\
\hline & -1.5 & $3(12)$ & $3(12)$ & $3(12)$ & $3(12)$ & $3(12)$ & $3(12)$ & $3(12)$ \\
\hline \multirow{4}{*}{$f_{7}$} & 1.0 & $2(8)$ & $2(8)$ & $3(12)$ & $3(12)$ & $3(12)$ & $3(12)$ & $2(8)$ \\
\hline & 0.8 & $3(12)$ & $\mathrm{NC}$ & $3(12)$ & $3(12)$ & $3(12)$ & $3(12)$ & $3(12)$ \\
\hline & 1.8 & $\mathrm{NC}$ & $\mathrm{NC}$ & $4(16)$ & $4(16)$ & NC & NC & $4(16)$ \\
\hline & 0.3 & $3(12)$ & $3(12)$ & $3(12)$ & $3(12)$ & $3(12)$ & $3(12)$ & $3(12)$ \\
\hline
\end{tabular}

Note: the choice of the parameters in the proposed method (19) is discussed in Section 4 based on the dynamical behavior for some test cases.

TABLE 4: Comparison of dynamical behavior of our proposed method (19) for different values of the parameters $\alpha, \beta, \gamma$, and $\delta$.

\begin{tabular}{|c|c|c|c|c|c|c|}
\hline Values of parameters & Method & $P_{1}(z)$ & $P_{2}(z)$ & $P_{3}(z)$ & $P_{4}(z)$ & Average \\
\hline$\alpha=1, \beta=-1, \gamma=1, \delta=1$ & $O M_{8,1}$ & 4 & 3 & 3 & 3 & $13 / 4$ \\
\hline$\alpha=-1, \beta=-1, \gamma=-1, \delta=-1$ & $O M_{8,2}$ & 3 & 3 & 4 & 3 & $13 / 4$ \\
\hline$\alpha=0, \beta=0, \gamma=3, \delta=0$ & $O M_{8,3}$ & 2 & 2 & 2 & 2 & $8 / 4$ \\
\hline$\alpha=0, \beta=3, \gamma=0, \delta=1$ & $O M_{8,4}$ & 1 & 1 & 1 & 1 & $4 / 4$ \\
\hline
\end{tabular}

By virtue of the above equation, we have

$$
\begin{aligned}
& +3 c_{1} c_{2}^{2}\left(-4 c_{3}+c_{1} A^{\prime \prime}(0)\right) \\
\times & \left(-20+3 B^{\prime \prime}(0)\right) \\
& +2 c_{1}^{2} c_{2}\left(-12 c_{4}+c_{1} A^{(3)}(0)\right) \\
& \left.+c_{2}^{4}\left(54\left(-4+B^{\prime \prime}(0)-2 B^{(3)}(0)\right)\right)\right) e_{n}^{5} \\
+\cdots+ & O\left(e_{n}^{9}\right) .
\end{aligned}
$$$$
f\left(z_{n}\right)=\frac{c_{2}\left(c_{1}\left(-2 c_{3}+c_{1} A^{\prime \prime}(0)\right)-c_{2}^{2}\left(-6+B^{\prime \prime}(0)\right)\right)}{2 c_{1}^{2}} e_{n}^{4}
$$$$
+\frac{1}{12 c_{1}^{3}}\left(-3 c_{1}^{2}\left(-4 c_{3}+c_{1} A^{\prime \prime}(0)\right)\right.
$$$$
\times\left(-2 c_{3}+c_{1} A^{\prime \prime}(0)\right)
$$ 


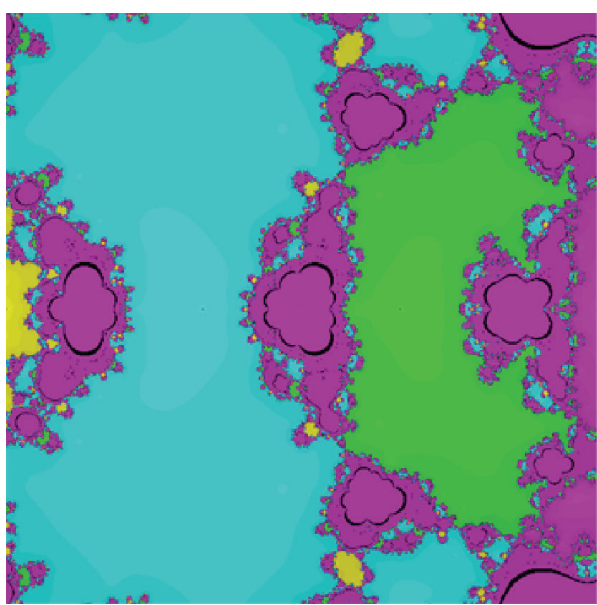

(a) $O M_{8,1}$

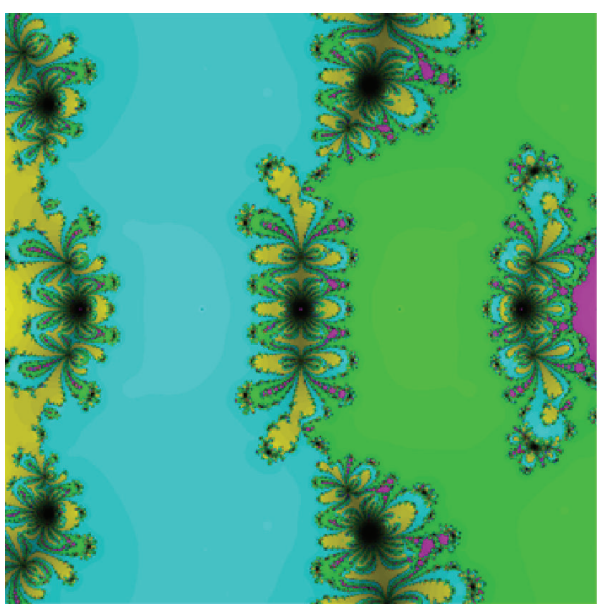

(c) $\mathrm{OM}_{8,3}$

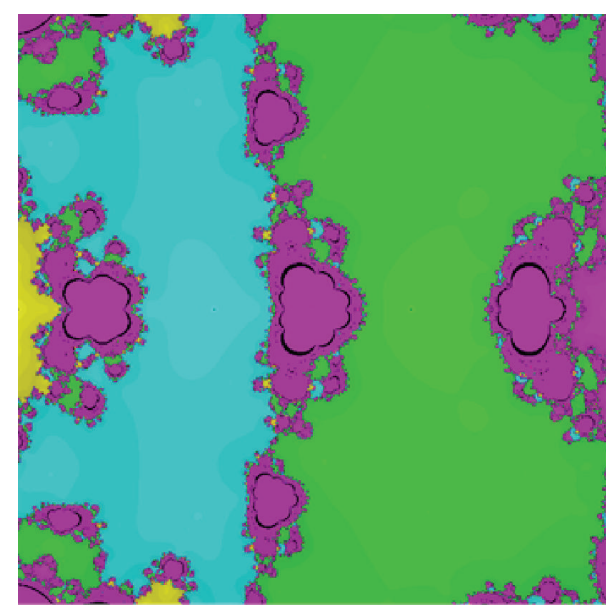

(b) $O M_{8,2}$

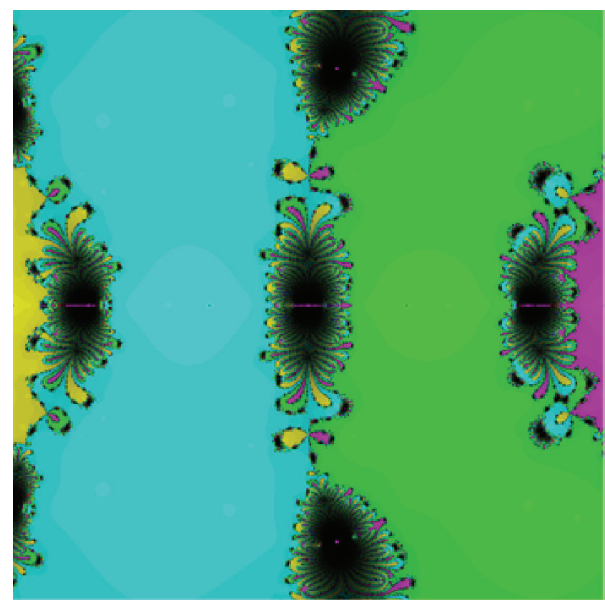

(d) $O M_{8,4}$

FigURE 1: Basins of attraction for polynomial $P_{1}(z)=\left(z^{4}-10 z^{2}+9\right)$.

With the help of the above expression, one can derive

$$
\begin{gathered}
f\left[y_{n}, z_{n}\right]=\frac{f\left(y_{n}\right)-f\left(z_{n}\right)}{y_{n}-z_{n}}=c_{1}+\frac{c_{2}^{2}}{c_{1}} e_{n}^{2}+\cdots+O\left(e_{n}^{9}\right) \\
\frac{f\left(z_{n}\right)}{f\left(y_{n}\right)}=\frac{c_{1}\left(-2 c_{3}+c_{1} A^{\prime \prime}(0)\right)-c_{2}^{2}\left(-6+B^{\prime \prime}(0)\right)}{2 c_{1}^{2}} e_{n}^{2} \\
+\frac{1}{6 c_{1}^{3}}\left\{3 c _ { 1 } c _ { 2 } \left(-4 c_{3}\left(-6+B^{\prime \prime}(0)\right)\right.\right. \\
\left.+c_{1} A^{\prime \prime}(0)\left(-5+B^{\prime \prime}(0)\right)\right) \\
+c_{1}^{2}\left(-12 c_{4}+c_{1} A^{(3)}(0)\right) \\
\left.+c_{2}^{3}\left(-72+21 B^{\prime \prime}(0)-B^{(3)}(0)\right)\right\} e_{n}^{3} \\
+\cdots+O\left(e_{n}^{9}\right)
\end{gathered}
$$

$$
\begin{gathered}
\frac{f\left(z_{n}\right)}{f\left(x_{n}\right)}=\frac{c_{2}\left(c_{1}\left(-2 c_{3}+c_{1} A^{\prime \prime}(0)\right)-c_{2}^{2}\left(-6+B^{\prime \prime}(0)\right)\right)}{2 c_{1}^{3}} e_{n}^{3} \\
+\frac{1}{12 c_{1}^{4}}\left\{-3 c_{1}^{2}\left(-4 c_{3}+c_{1} A^{\prime \prime}(0)\right)\left(-2 c_{3}+c_{1} A^{\prime \prime}(0)\right)\right. \\
+3 c_{1} c_{2}^{2}\left(-12 c_{3}\left(-7+B^{\prime \prime}(0)\right)\right. \\
\left.+c_{1} A^{\prime \prime}(0)\left(-22+3 B^{\prime \prime}(0)\right)\right) \\
+2 c_{1}^{2} c_{2}\left(-12 c_{4}+c_{1} A^{(3)}(0)\right) \\
\left.+c_{2}^{4}\left(60 B^{\prime \prime}(0)-2\left(126+B^{(3)}(0)\right)\right)\right\} e_{n}^{4} \\
+\cdots+O\left(e_{n}^{9}\right)
\end{gathered}
$$

Finally, using (10), (15), (16), (12), (13), (14) and $R(0)=1-$ $P(0)-Q(0), P^{\prime}(0)=0, Q^{\prime}(0)=0, P^{\prime \prime}(0)=2, R^{\prime}(0)=2$, 


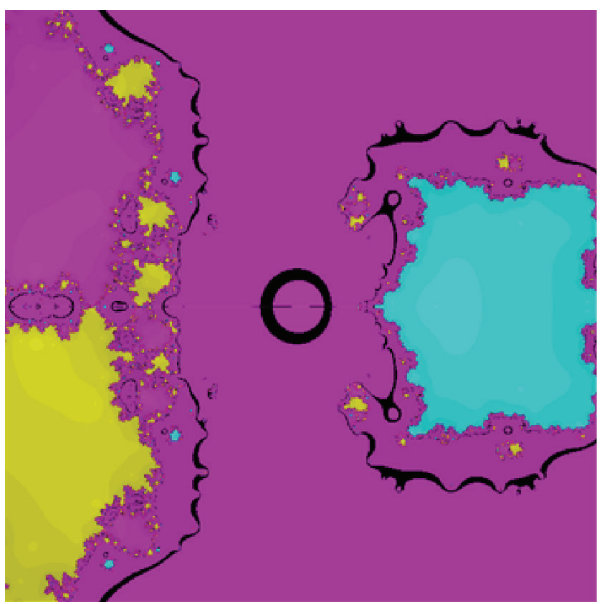

(a) $O M_{8,1}$

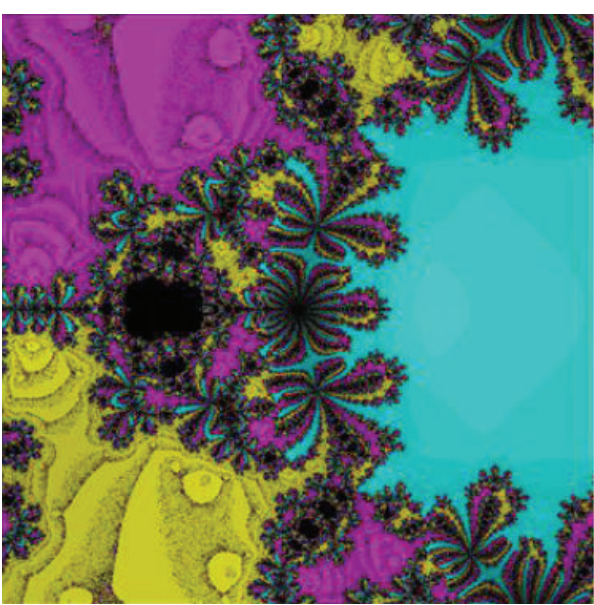

(c) $\mathrm{OM}_{8,3}$

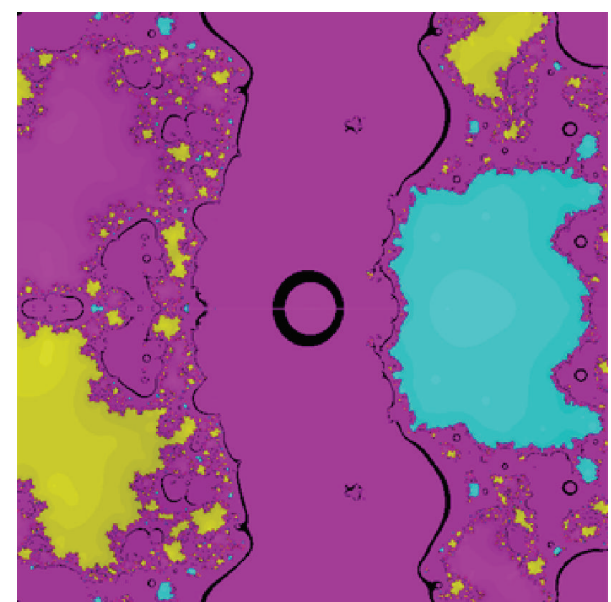

(b) $O M_{8,2}$

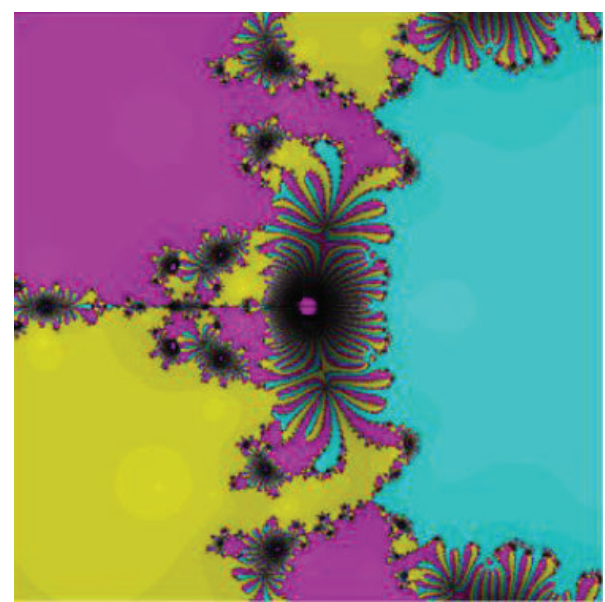

(d) $O M_{8,4}$

Figure 2: Basins of attraction for polynomial $P_{2}(z)=\left(z^{3}+4 z^{2}-10\right)$.

$A^{\prime \prime}(0)=0, P^{(3)}(0)=6 B^{\prime \prime}(0)-12$, in the last step of (3), we get the final error expression which is given by

$$
\begin{aligned}
e_{n+1}= & \frac{c_{2}}{48 c_{1}^{7}}\left\{2 c_{1} c_{3}+c_{2}^{2}\left(-6+B^{\prime \prime}(0)\right)\right\} \\
\times & \left\{12 c_{1}^{2} c_{3}^{2} Q^{\prime \prime}(0)+12 c_{1} c_{2}^{2} c_{3}\right. \\
& \times\left(8+\left(-6+B^{\prime \prime}(0)\right) Q^{\prime \prime}(0)\right) \\
& +4 c_{1}^{2} c_{2}\left(-6 c_{4}+c_{1} A^{(3)}(0)\right) \\
+ & c_{2}^{4}\left(108 Q^{\prime \prime}(0)+3 B^{\prime \prime}(0)\right. \\
& \times\left(8+\left(-12+B^{\prime \prime}(0)\right) Q^{\prime \prime}(0)\right) \\
& \left.\left.\quad-8\left(9+B^{(3)}(0)+P^{(4)}(0)\right)\right)\right\} e_{n}^{8}+O\left(e_{n}^{9}\right) .
\end{aligned}
$$

Thus, theorem is proved.
Particular Case. Let

$$
\begin{gathered}
A\left(t_{1}\right)=1+\alpha t_{1}^{3}, \\
B\left(t_{2}\right)=1+t_{2}+\beta t_{2}^{2}, \\
P\left(t_{2}\right)=t_{2}^{2}+2(\beta-1) t_{2}^{3}, \\
Q\left(t_{3}\right)=\gamma t_{3}^{2}, \\
R\left(t_{4}\right)=1+2 t_{4}+\delta t_{4}^{2},
\end{gathered}
$$

where $\alpha, \beta, \gamma$, and $\delta \in \mathbb{R}$; then the method becomes

$$
y_{n}=x_{n}-\left\{1+\alpha t_{1}^{3}\right\} \cdot \frac{f\left(x_{n}\right)}{f^{\prime}\left(x_{n}\right)},
$$

$$
z_{n}=y_{n}-\left\{1+t_{2}+\beta t_{2}^{2}\right\} \cdot \frac{f\left(y_{n}\right)}{f\left[x_{n}, y_{n}\right]} \text {, }
$$




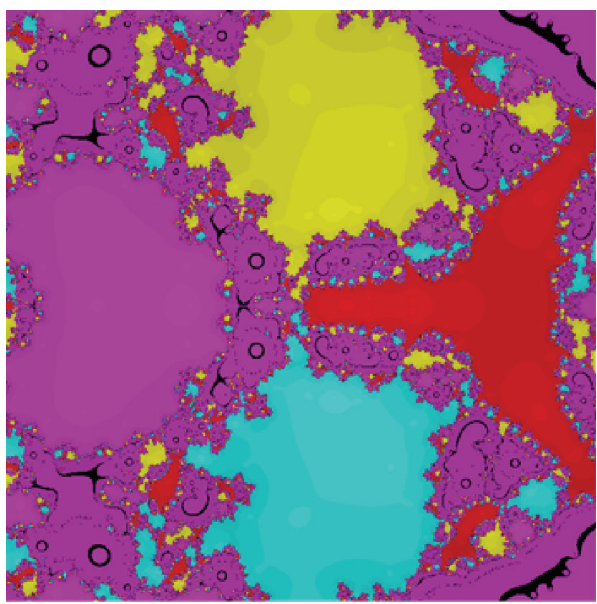

(a) $O M_{8,1}$

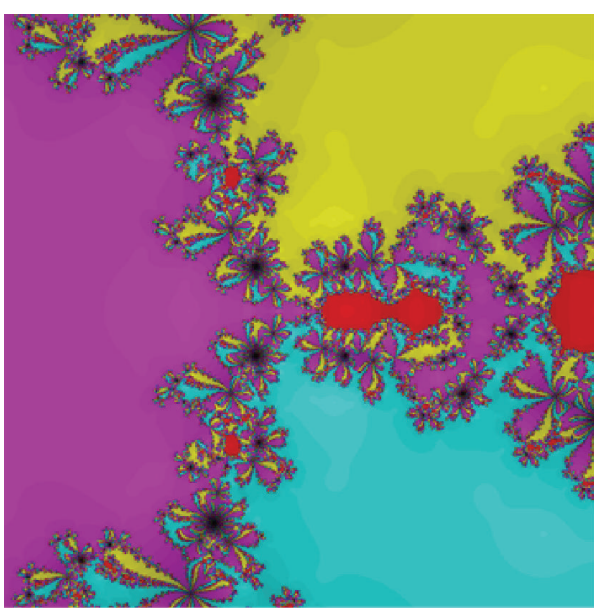

(c) $O M_{8,3}$

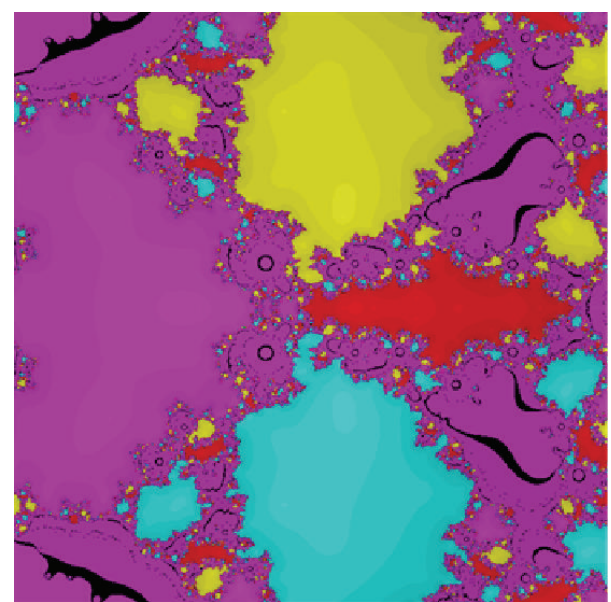

(b) $O M_{8,2}$

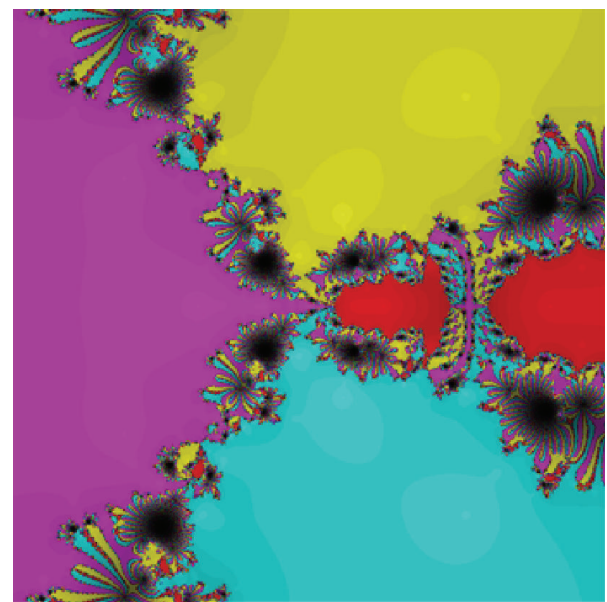

(d) $O M_{8,4}$

FIGURE 3: Basins of attraction for non-polynomial $P_{3}(z)=\left(z^{3}-1 / 2 z+1\right)$.

$$
\begin{aligned}
x_{n+1}=z_{n}-\{ & \left\{t_{2}^{2}+2(\beta-1) t_{2}^{3}\right\}+\left\{\gamma t_{3}^{2}\right\} \\
+ & \left.\left\{1+2 t_{4}+\delta t_{4}^{2}\right\}\right\} \cdot \frac{f\left(z_{n}\right)}{f\left[y_{n}, z_{n}\right]},
\end{aligned}
$$

where $t_{1}=f\left(x_{n}\right) / f^{\prime}\left(x_{n}\right), t_{2}=f\left(y_{n}\right) / f\left(x_{n}\right), t_{3}=f\left(z_{n}\right) /$ $f\left(y_{n}\right)$, and $t_{4}=f\left(z_{n}\right) / f\left(x_{n}\right)$. Then its error expression becomes

$$
\begin{aligned}
e_{n+1}= & \frac{c_{2}}{c_{1}^{7}}\left\{c_{1} c_{3}+c_{2}^{2}(-3+\beta)\right\} \\
& \cdot\left\{\alpha c_{1}^{3} c_{2}+\left(-3+2 \beta+\left(-3+\beta^{2}\right) \gamma\right) c_{2}^{4}\right. \\
& \left.+2(2+(-3+\beta) \gamma) c_{1} c_{2}^{2} c_{3}+c_{1}^{2}\left(\gamma c_{3}^{2}-c_{2} c_{4}\right)\right\} \\
& \times e_{n}^{8}+O\left(e_{n}^{9}\right) .
\end{aligned}
$$

Remark 2. By taking different values of $\alpha, \beta, \gamma$, and $\delta$ one can get a number of eighth-order iterative methods. Clearly its efficiency index is $8^{1 / 4} \approx 1.682$ which is more than $7^{1 / 4} \approx$ 1.626 of the method (2).

\section{Results and Discussion}

This section deals with the numerical comparisons of the proposed method (19). We have taken four particular cases of the proposed method (19) by considering different values of $\alpha, \beta, \gamma$, and $\delta$. The result of the comparisons of these methods based on the dynamical behavior is given in Table 4 . From Table 4, we found that for $\alpha=\gamma=0, \beta=3$, and $\delta=1$ method $O M_{8,4}$ is more efficient than the other ones. In order to check the effectiveness of the proposed iterative method $O M_{8,4}$ we have considered seven test nonlinear functions which are taken from [10]. The test nonlinear functions and their roots are listed in Table 1. In recent days, higher-order methods are very important because numerical applications use high precision computations. Due to this reason all the computations reported have been performed on the programming package MATHEMATICA8 using 1000 digits floating point arithmetic using "SetAccuraccy" command. The results of 


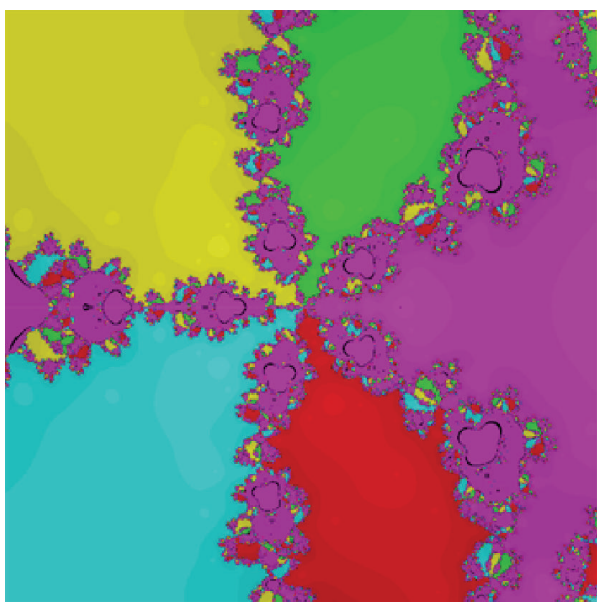

(a) $O M_{8,1}$

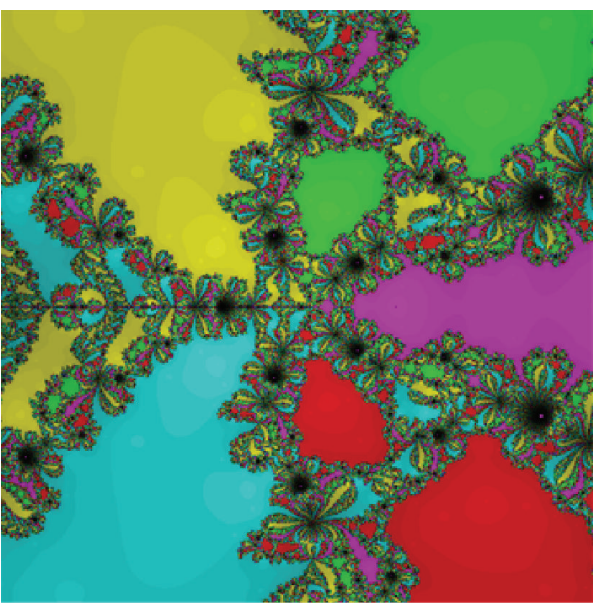

(c) $O M_{8,3}$

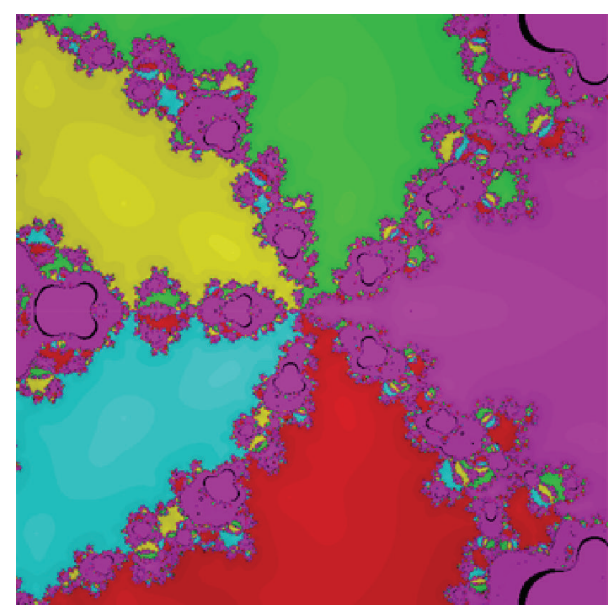

(b) $O M_{8,2}$

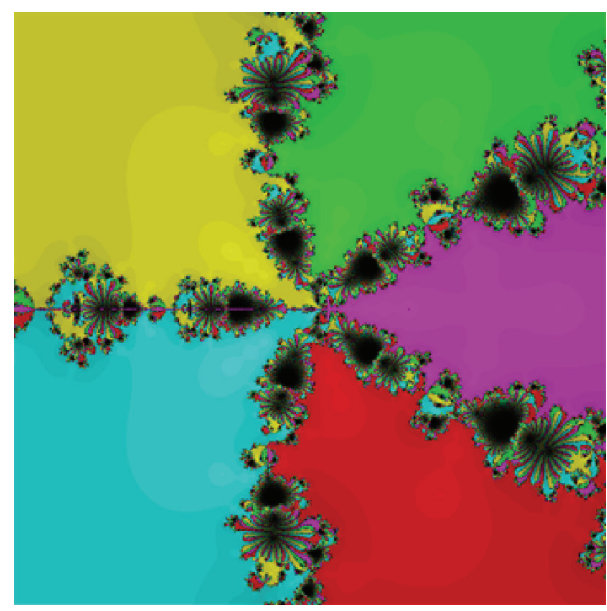

(d) $\mathrm{OM}_{8,4}$

FIGURE 4: Basins of attraction for nonpolynomial $P_{4}(z)=\left(z^{4}-1 / z\right)$.

the comparisons are given in Tables 2 and 3. The Computer characteristics during numerical calculations are Microsoft Windows 8 Intel Core i5-3210M CPU@2.50 GHz with 4.00 GB of RAM, 64-bit Operating System throughout this paper. Here, we compare the performance of our new eighthorder method $\left(\mathrm{OM}_{8,4}\right)$ with the methods (34) $\left(M_{8,1}\right)$, (35) $\left(M_{8,2}\right)$ of [11]; NM2 $\left(M_{8,3}\right)$, NM3 $\left(M_{8,4}\right)$ of [12]; (11) $\left(M_{8,5}\right)$, (15) $\left(M_{8,6}\right)$ of [10]. Table 2 represents the value of $\left|f\left(x_{n}\right)\right|$ calculated for the total number of function evaluations twelve (TNFE-12) for each scheme. Table 3 exhibits the number of iterations and total number of function evaluations using the stopping criteria $\left|f\left(x_{n+1}\right)\right|<\epsilon$ where $\epsilon=10^{-50}$. It can be observed from Tables 2 and 3 that our method $O M_{8,4}$ is competitive.

\section{Dynamical Analysis}

In this section, we study the dynamic comparison of some higher-order simple root methods in the complex plane using basins of attraction. The basin of attraction is a way to view how an algorithm behaves as a function of the various starting point. It is an another way to compare the iterative methods. Recently, different iterative methods have been compared by their basins of attraction in [13-17]. In order to compare these methods we consider a rectangle $D=[-3,3] \times[-3,3] \in \mathbb{C}$ and assign a color to each point $z_{0} \in D$, according to the root at which the corresponding iterative method starting from $z_{0}$ converges, and we mark the point as black if the method does not converge. We have used stopping criteria $10^{-4}$ and maximum number of iterations 200 for each method. In this section, the following test polynomials and nonpolynomials have been considered for comparison:
(i) $P_{1}(z)=\left(z^{4}-10 z^{2}+9\right)$
(ii) $P_{2}(z)=\left(z^{3}+4 z^{2}-10\right)$
(iii) $P_{3}(z)=\left(z^{3}-1 / 2 z+1\right)$
(iv) $P_{4}(z)=\left(z^{4}-1 / z\right)$.

Here, we try to find the optimal value of the parameters used in the proposed method (19). In order to obtain the optimal value, we have given rank 1 for best and rank 4 for the worst performance in each polynomial. The average value of 


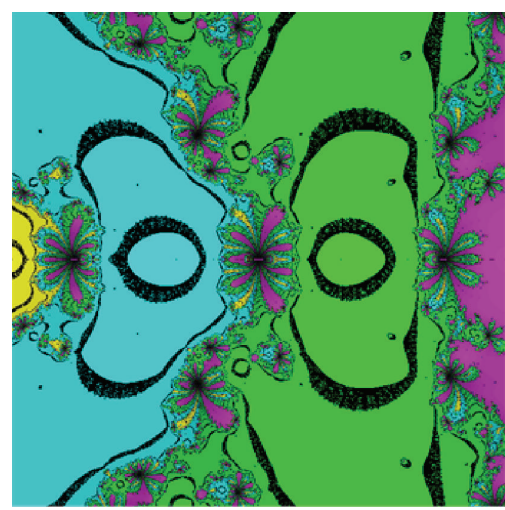

(a) $M_{8,1}$

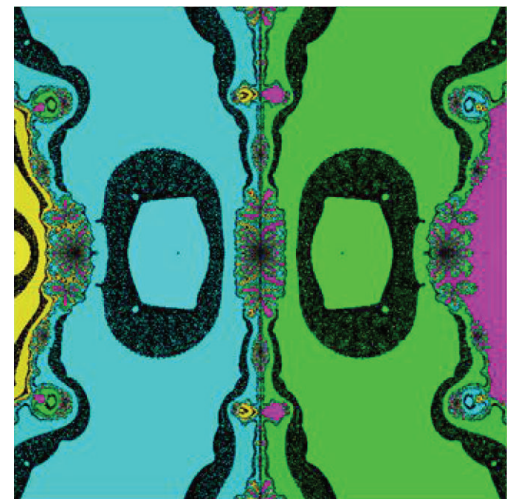

(c) $M_{8,3}$

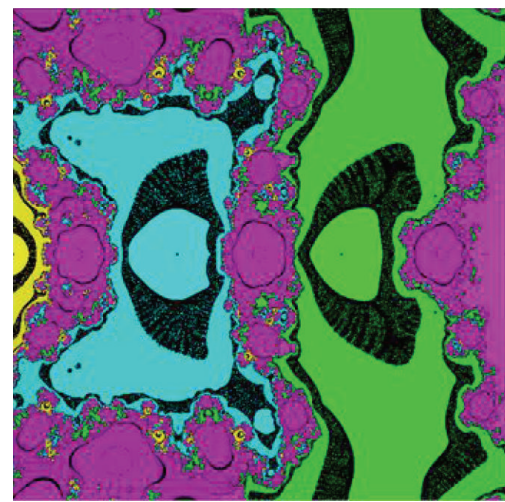

(e) $M_{8,5}$

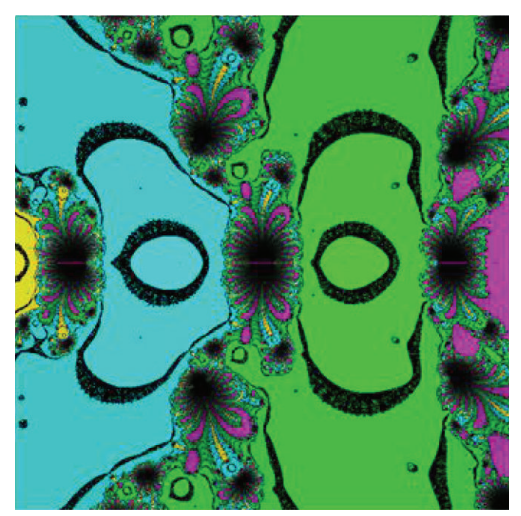

(b) $M_{8,2}$

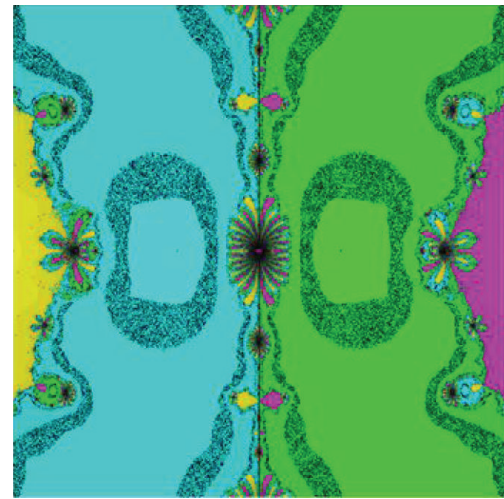

(d) $M_{8,4}$

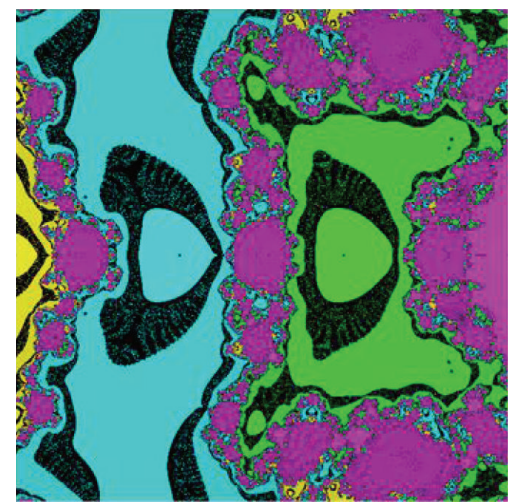

(f) $M_{8,6}$

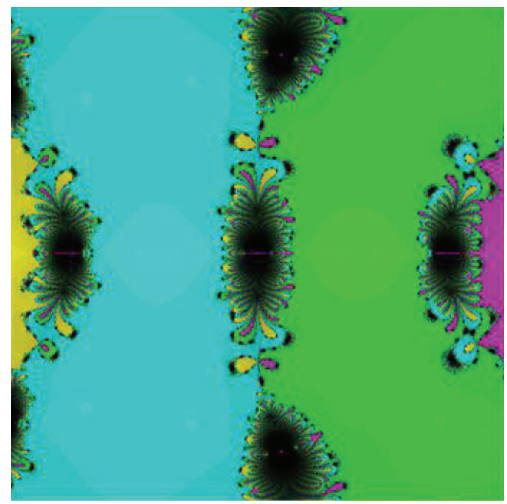

(g) $O M_{8,4}$

FIGURE 5: Basins of attraction for polynomial $P_{1}(z)=\left(z^{4}-10 z^{2}+9\right)$. 


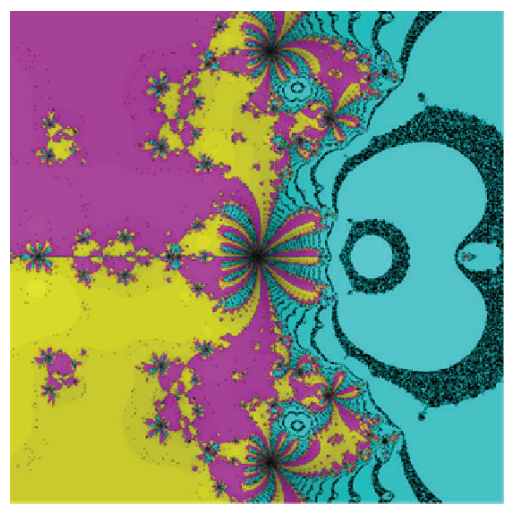

(a) $M_{8,1}$

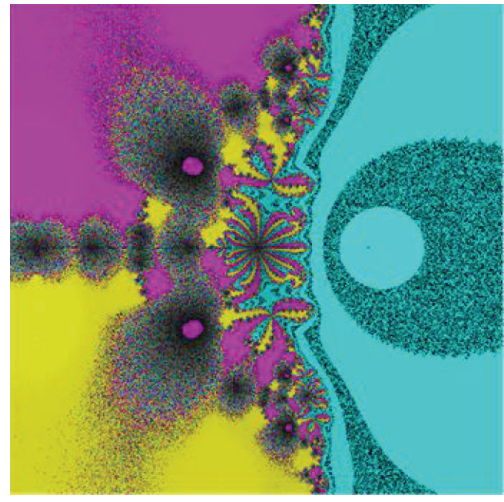

(c) $M_{8,3}$

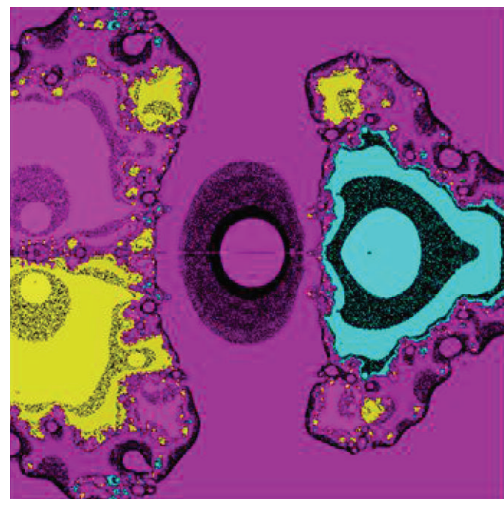

(e) $M_{8,5}$

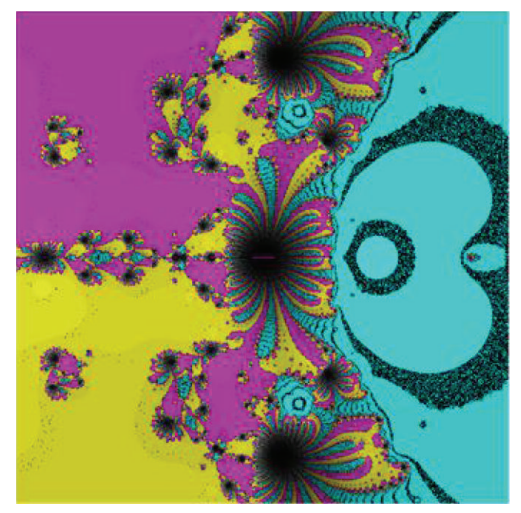

(b) $M_{8,2}$

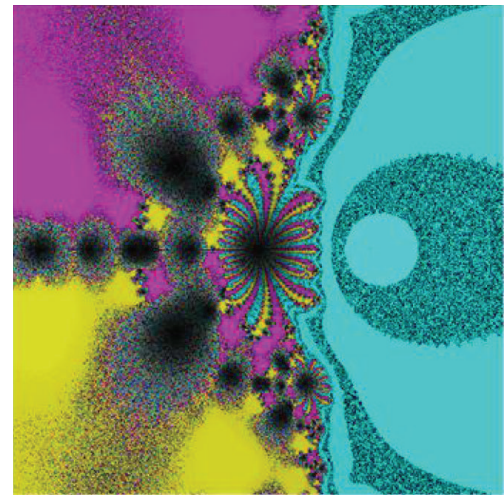

(d) $M_{8,4}$

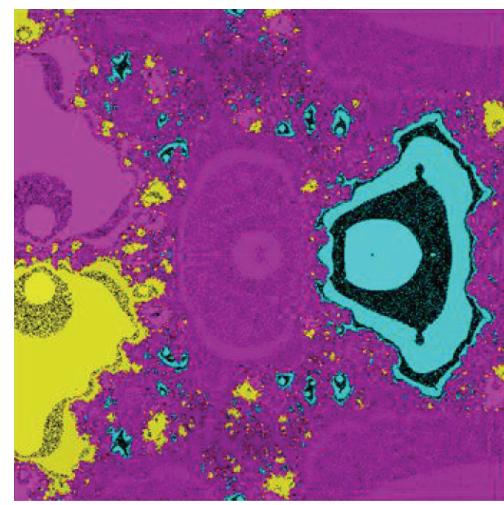

(f) $M_{8,6}$

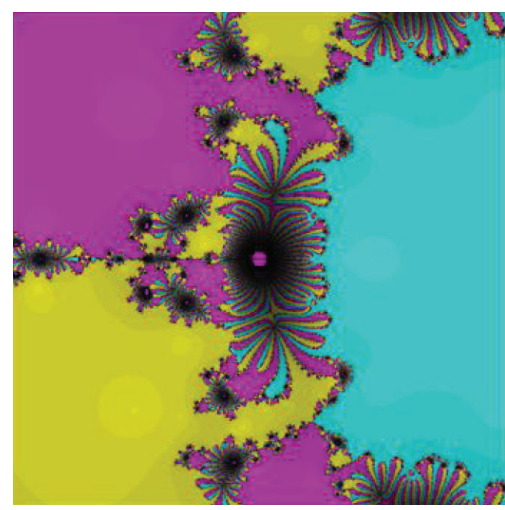

(g) $O M_{8,4}$

Figure 6: Basins of attraction for polynomial $P_{2}(z)=\left(z^{3}+4 z^{2}-10\right)$. 


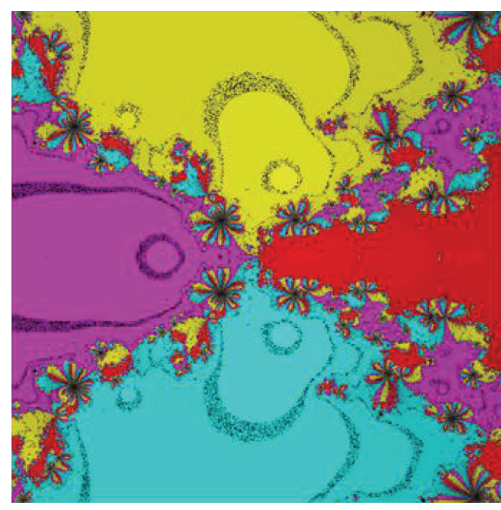

(a) $M_{8,1}$

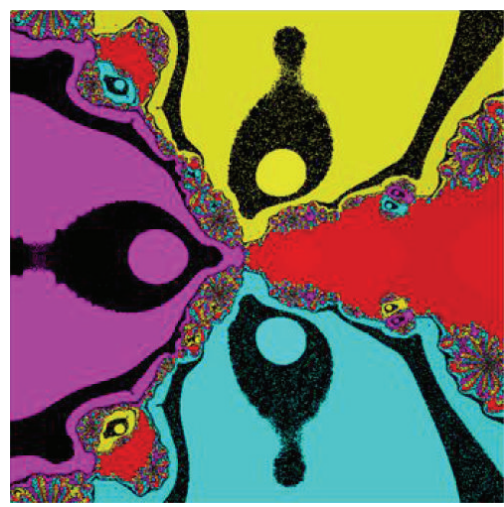

(c) $M_{8,3}$

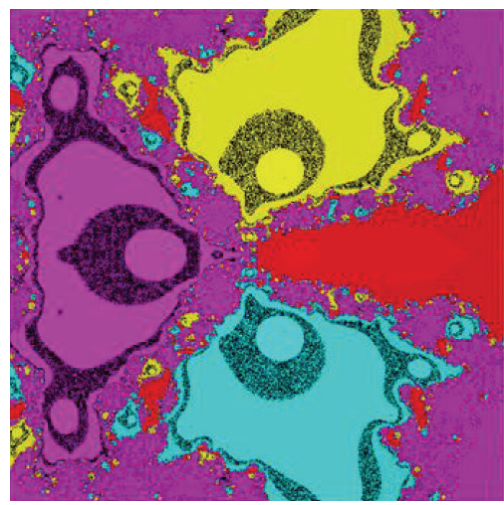

(e) $M_{8,5}$

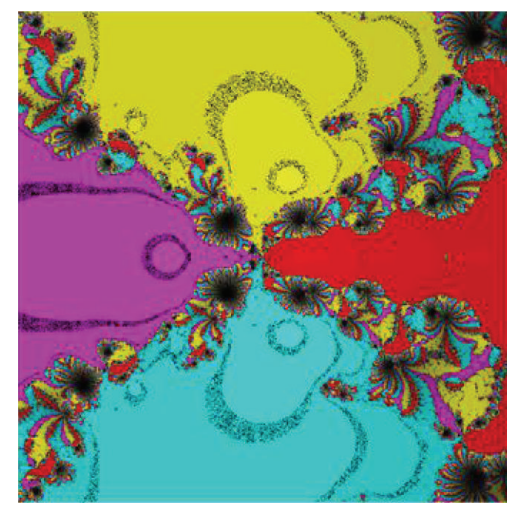

(b) $M_{8,2}$

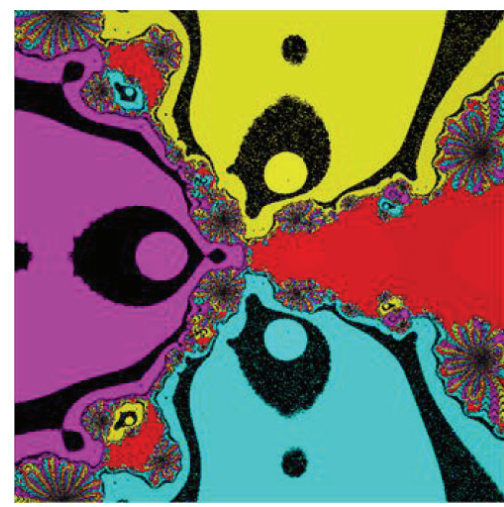

(d) $M_{8,4}$

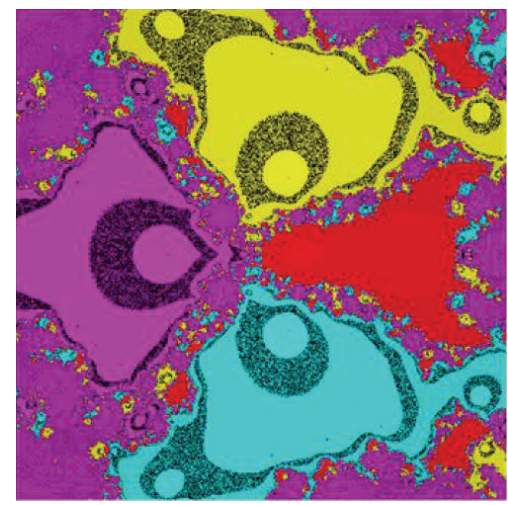

(f) $M_{8,6}$

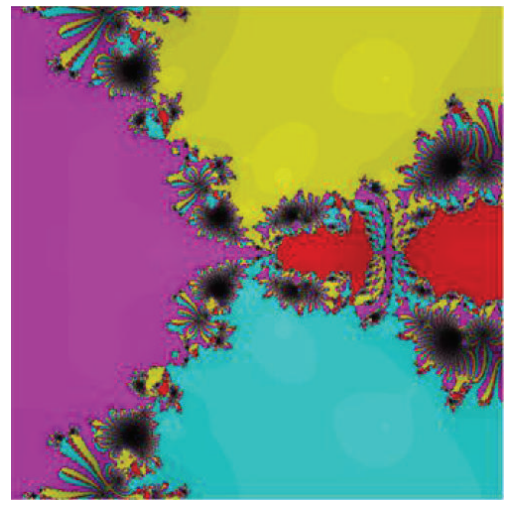

(g) $O M_{8,4}$

FIGURE 7: Basins of attraction for nonpolynomial $P_{3}(z)=\left(z^{3}-1 / 2 z+1\right)$. 


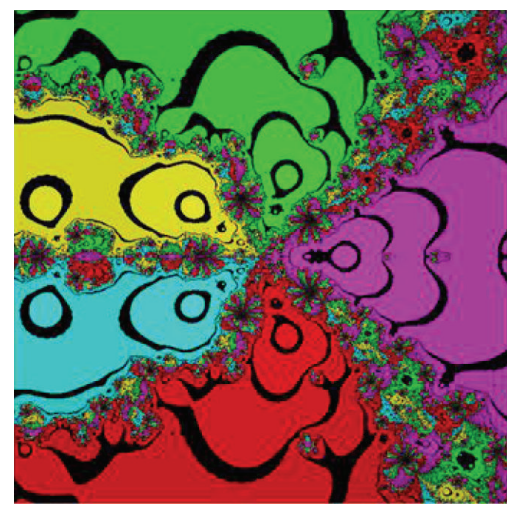

(a) $M_{8,1}$

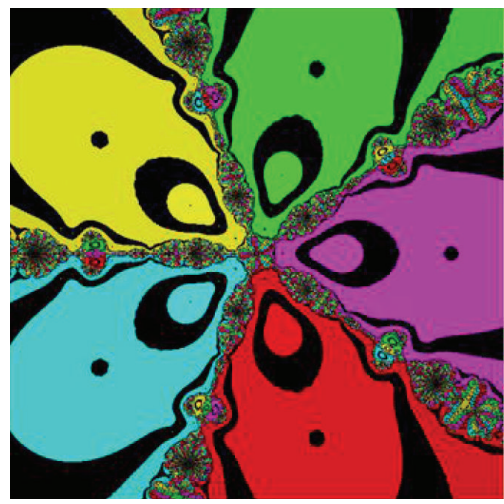

(c) $M_{8,3}$

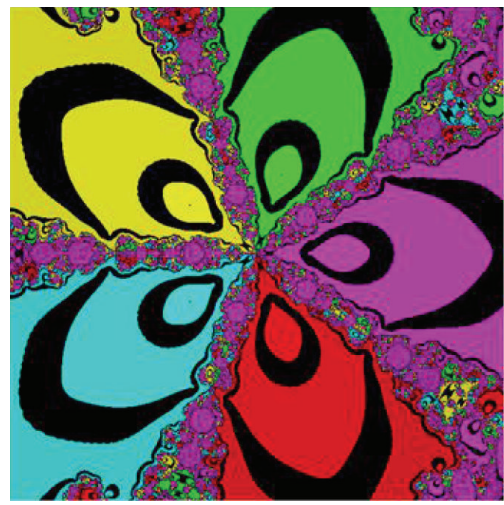

(e) $M_{8,5}$

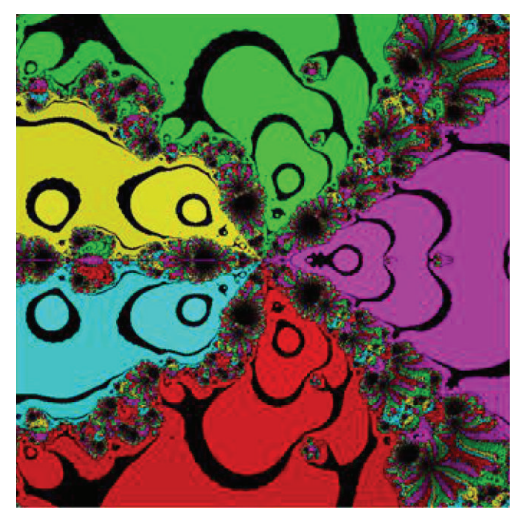

(b) $M_{8,2}$

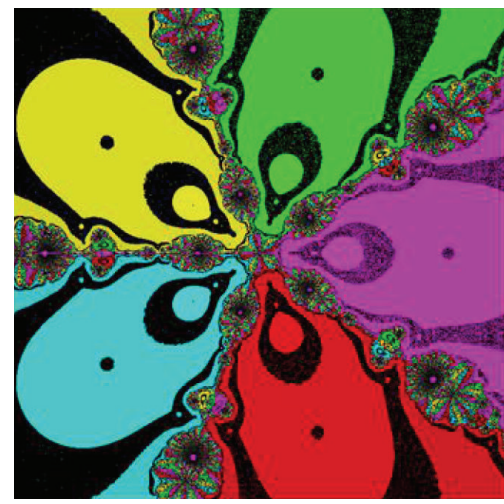

(d) $M_{8,4}$

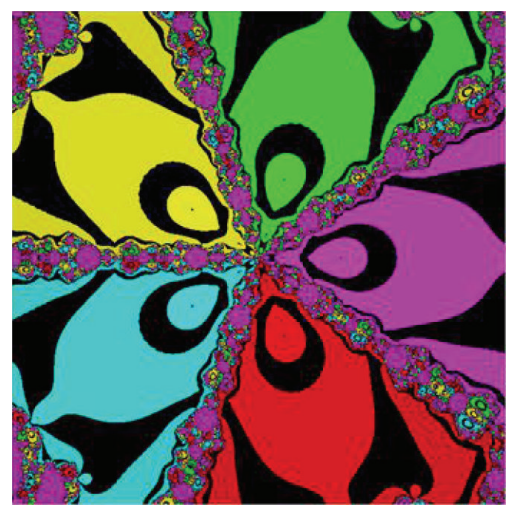

(f) $M_{8,6}$

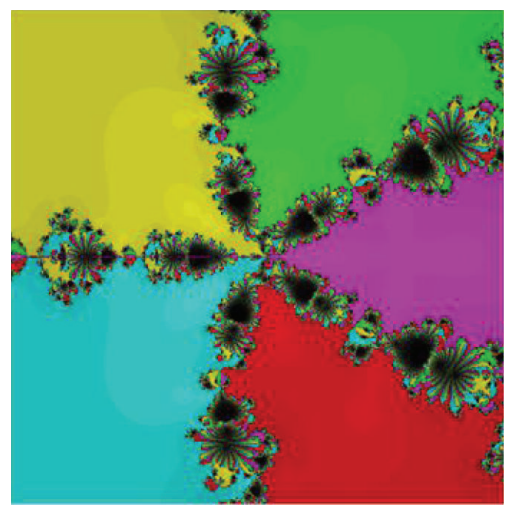

(g) $O M_{8,4}$

FIGURE 8: Basins of attraction for nonpolynomial $P_{4}(z)=\left(z^{4}-1 / z\right)$. 
the ranks for each method has been mentioned in Table 4. The method with least average value will be the best performer. In Figure 1, it can be easily observed that for polynomial (i) the dynamical behavior of the method $O M_{8,4}$ is superior than the methods $O M_{8,1}, O M_{8,2}$, and $O M_{8,3}$. So, we have given rank 1 to method $O M_{8,4}$ and rank 2 to method $O M_{8,3}$. The method $O M_{8,2}$ is slightly better than $O M_{8,1}$ so we have assigned rank 3 to method $O M_{8,2}$ and rank 4 to method $O M_{8,1}$.

Based on Figure 2, for polynomial (ii) again $O M_{8,4}$ is best, followed by method $O M_{8,3}$. The methods $O M_{8,4}$ and $O M_{8,3}$ have given the rank 1 and 2, respectively. The behavior of methods $O M_{8,1}$ and $O M_{8,2}$ is almost similar with rank 3 .

According to Figure 3, $O M_{8,4}$ and $O M_{8,3}$ have been given the ranks 1 and 2, respectively, for nonpolynomial (iii). Methods $O M_{8,1}$ and $O M_{8,2}$ are given the ranks 3 and 4, respectively.

From Figure 4, it can be seen that again $O M_{8,4}$ is best with rank 1. Method $O M_{8,3}$ has rank 2. The dynamical behaviors of methods $O M_{8,1}$ and $O M_{8,2}$ have rank 3 due to their almost similar basins of attraction.

Finally, from Table 4 we observe that the average rank for the method $O M_{8,4}$ is minimum. The method $O M_{8,4}$ is better than others in terms of less chaotic behavior and large basins of attraction for finding the solutions. Hence, we will consider it for comparison with some other well established existing eighth-order methods.

Polynomial (i), has roots $3,-3,-1$, and 1 . According to Figure 5, we found that $O M_{8,4}$ is best and other methods $M_{8,3}, M_{8,4}, M_{8,1}$, and $M_{8,2}$ do not perform well. Methods $M_{8,5}$ and $M_{8,6}$ show chaotic behavior. Polynomial (ii) has roots $-2.68262+0.358259 I,-2.68262-0.358259 I$, and 1.36523 . According to Figure 6, we found that $O M_{8,4}$ is best and other methods $M_{8,3}, M_{8,4}, M_{8,1}$, and $M_{8,2}$ do not perform well. Again methods $M_{8,5}$ and $M_{8,6}$ show chaotic behavior.

Nonpolynomial (iii) has roots $-1.1299,0.336674+$ $0.925153 I, 0.336674-0.925153 I$, and 0.456553 . Based on Figure 7, we observe that method $O M_{8,4}$ dominates other methods.

Nonpolynomial (iv) has roots $1,-0.809017+0.587785 I$, $-0.809017-0.587785 I, 0.309017+0.951057 I$, and $0.309017-$ 0.951057I. In Figure 8, again $O M_{8,4}$ performs better than other methods. Methods $M_{8,1}, M_{8,2}, M_{8,3}, M_{8,4}, M_{8,5}$, and $M_{8,6}$ have large number of diverging points (black points).

In this section, we observe that some schemes have simple boundary of basins and other have complex boundary of basins. We also found that some existing iterative methods have chaotic behavior. Based of Figures 5, 6, 7, and 8 we also observe that methods $M_{8,1}, M_{8,2}, M_{8,3}, M_{8,4}, M_{8,5}$, and $M_{8,6}$ have more diverging points (black area) in comparison with $O M_{8,4}$. Finally, our method $O M_{8,4}$ has less number of diverging points and large basins of attraction.

\section{Conclusion}

In this paper, we have developed a new family of optimal eighth-order iterative methods to find simple root of nonlinear equations. New proposed family is obtained by improving the order of convergence of the existing seventhorder method without adding more evaluations and using the weight function technique. Numerical and dynamical comparisons have also presented to show the performance of the new method. From numerical and graphical comparisons, we can conclude that the new family is efficient and give tough competition to some existing eighth-order methods.

\section{Conflict of Interests}

The authors declare that there is no conflict of interests regarding the publication of this paper.

\section{Acknowledgment}

The authors are highly grateful to the reviewers for their precious suggestions, in improving the quality and presentation of the original paper.

\section{References}

[1] S. Artidiello, A. Cordero, J. R. Torregrosa, and M. P. Vassileva, "Two weighted eight-order classes of iterative root-finding methods," International Journal of Computer Mathematics, 2014.

[2] C. Chun and M. Y. Lee, "A new optimal eighth-order family of iterative methods for the solution of nonlinear equations," Applied Mathematics and Computation, vol. 223, pp. 506-519, 2013.

[3] A. Cordero, T. Lotfi, K. Mahdiani, and J. R. Torregrosa, "Two optimal general classes of iterative methods with eight-order," Acta Applicandae Mathematicae, pp. 1-14, 2014.

[4] H. T. Kung and J. F. Traub, "Optimal order of one-point and multipoint iteration," Journal of the Association for Computing Machinery, vol. 21, pp. 643-651, 1974.

[5] M. S. Petkovic, B. Neta, L. D. Petkovic, and J. Dzunic, Multipoint Methods for Solving Nonlinear Equations, Elsevier, 2012.

[6] F. Soleymani, "New class of eighth-order iterative zero-finders and their basins of attraction," Afrika Matematika, vol. 25, no. 1, pp. 67-79, 2014.

[7] J. F. Traub, Iterative Methods for Solution of Equations, Chelsea Publishing, New York, NY, USA, 2nd edition, 1982.

[8] C. Chun, B. Neta, J. Kozdon, and M. Scott, "Choosing weight functions in iterative methods for simple roots," Applied Mathematics and Computation, vol. 227, pp. 788-800, 2014.

[9] F. Soleymani and B. S. Mousavi, "On novel classes of iterative methods for solving nonlinear equations," Computational Mathematics and Mathematical Physics, vol. 52, no. 2, pp. 203210, 2012.

[10] D. K. R. Babajee, A. Cordero, F. Soleymani, and J. R. Torregrosa, "On improved three-step schemes with high efficiency index and their dynamics," Numerical Algorithms, vol. 65, no. 1, pp. 153-169, 2014.

[11] T. Lotfi, A. Cordero, J. R. Torregrosa, M. Amir Abadi, and M. Mohammadi Zadeh, "On generalization based on Bi et al. iterative methods with eighth-order convergence for solving nonlinear equations," The Scientific World Journal, vol. 2014, Article ID 272949, 8 pages, 2014.

[12] J. R. Sharma and H. Arora, "An efficient family of weightednewton methods with optimal eighth order convergence," Applied Mathematics Letters, vol. 29, pp. 1-6, 2014.

[13] A. Cordero, F. Soleymani, J. R. Torregrosa, and S. Shateyi, "Basins of attraction for various Steffensen-type methods," 
Journal of Applied Mathematics, vol. 2014, Article ID 539707, 17 pages, 2014.

[14] B. Neta, M. Scott, and C. Chun, "Basins of attraction for several methods to find simple roots of nonlinear equations," Applied Mathematics and Computation, vol. 218, no. 21, pp. 10548-10556, 2012.

[15] B. Neta and C. Chun, "Basins of attraction for several optimal fourth order methods for multiple roots," Mathematics and Computers in Simulation, vol. 103, pp. 39-59, 2014.

[16] M. Scott, B. Neta, and C. Chun, "Basin attractors for various methods," Applied Mathematics and Computation, vol. 218, no. 6, pp. 2584-2599, 2011.

[17] J. L. Varona, "Graphic and numerical comparison between iterative methods," The Mathematical Intelligencer, vol. 24, no. 1, pp. 37-46, 2002. 


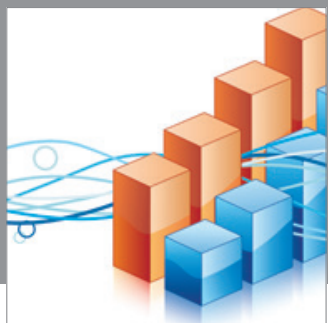

Advances in

Operations Research

mansans

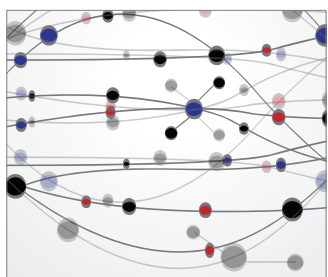

The Scientific World Journal
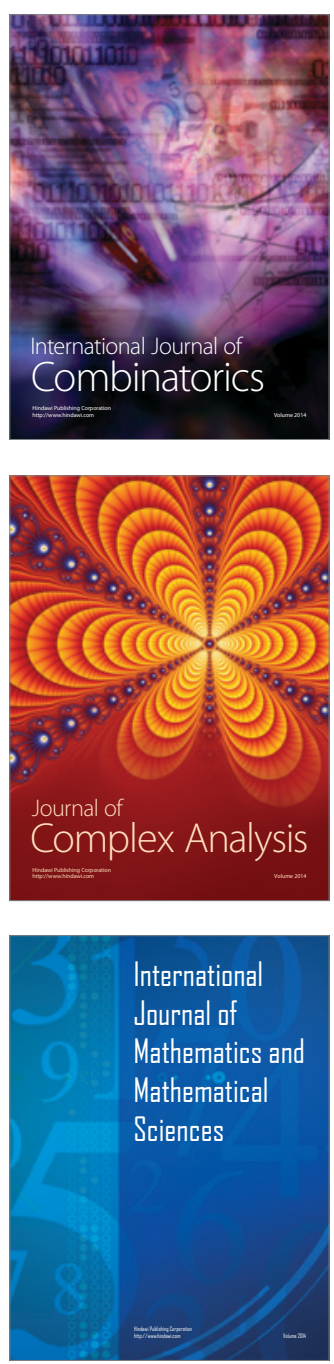
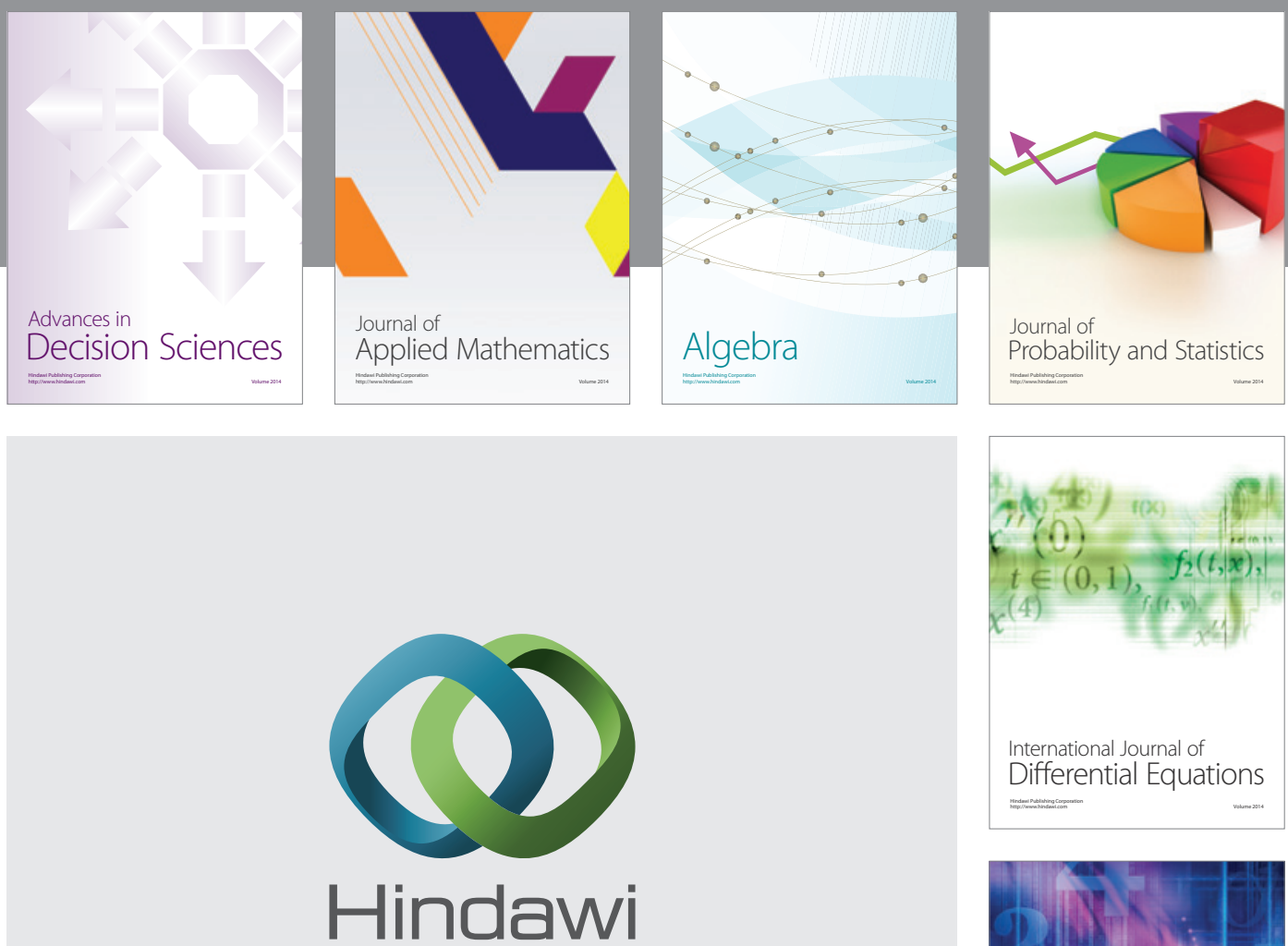

Submit your manuscripts at http://www.hindawi.com
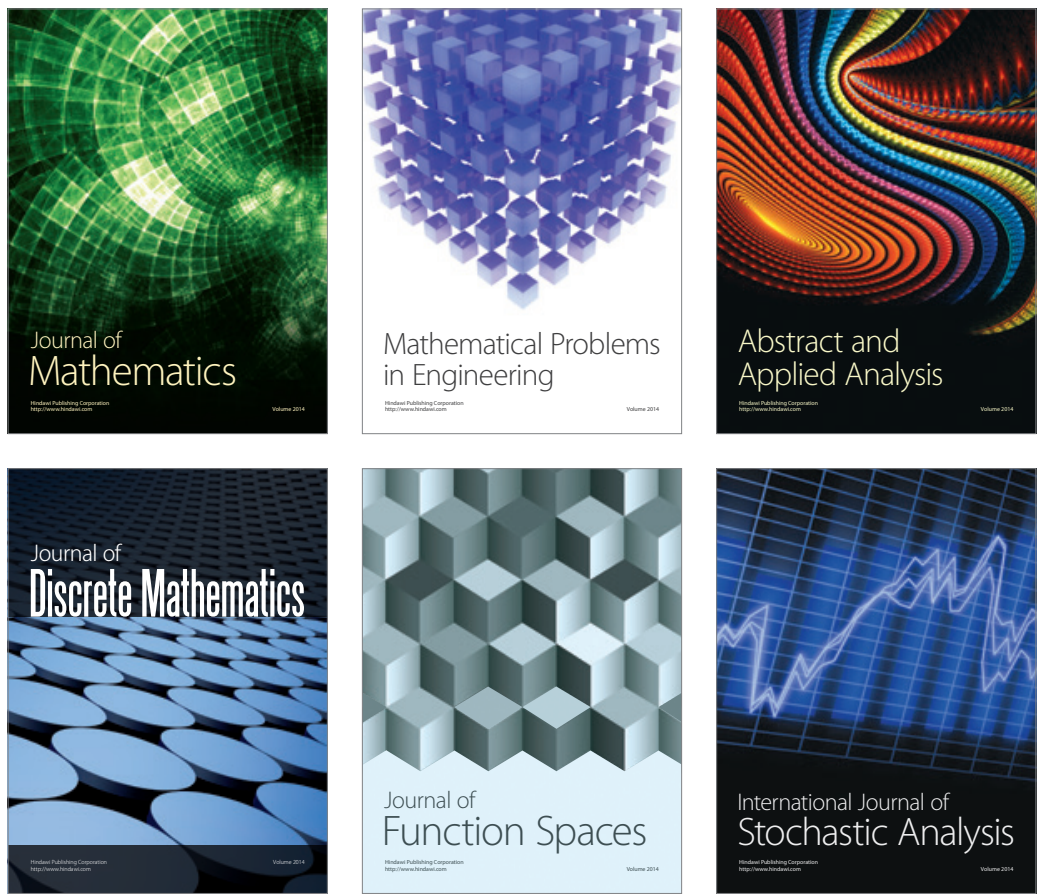

Journal of

Function Spaces

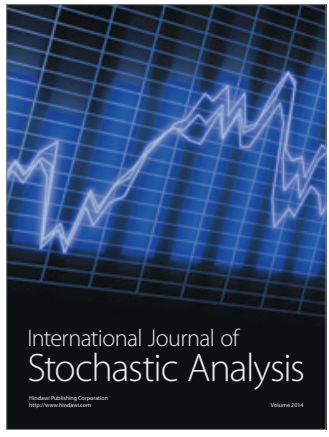

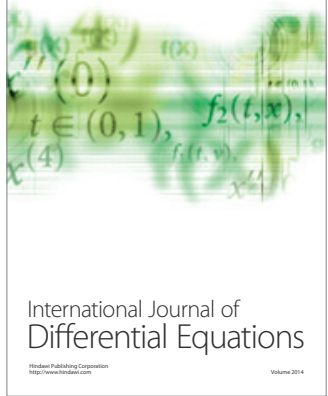
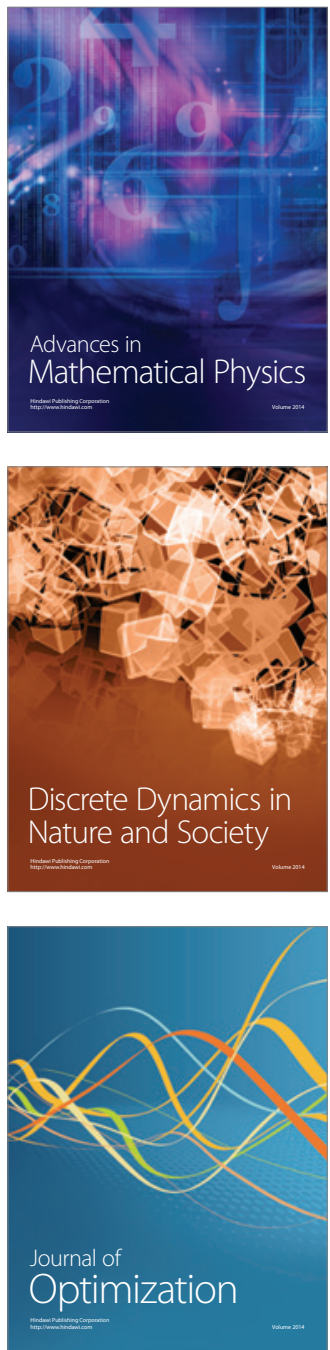